\title{
Stroke Bricks - spatial brain regions to assess ischaemic stroke localisation
}

\author{
B. Ciszek ${ }^{1,2}$, R. Jóźwiak³ ${ }^{3}$ E. Sobieszczuk ${ }^{4}$, A. Przelaskowski ${ }^{3}$ T. Skadorwa1,2 \\ ${ }^{1}$ Department of Descriptive and Clinical Anatomy, Centre of Biostructure Research, \\ Medical University of Warsaw, Poland \\ 2Department of Neurosurgery, Bogdanowicz Memorial Hospital, Warsaw, Poland \\ ${ }^{3}$ Faculty of Mathematics and Information Science, Warsaw University of Technology, Warsaw, Poland \\ ${ }^{4}$ Department of Neurology, Medical University of Warsaw, Poland
}

[Received: 30 December 2016; Accepted: 7 February 2017]

\begin{abstract}
Computer-aided analysis of non-contrast computed tomography (NCCT) images for rapid diagnosis of ischaemic stroke is based on the augmented visualisation of evolving ischaemic lesions. Computerised support of NCCT often leads to overinterpretation of ischaemic areas, thus it is of great interest to provide neurologically verified regions in order to improve accuracy of subsequent radiological assessment.

We propose Stroke Bricks (StBr) as an arbitrary spatial division of brain tissue into the regions associated with specific clinical symptoms of ischaemic stroke. Neurological stroke deficit is formally translated into respective areas of possible ischaemic lesions. StBr were designed according to formalised mapping of neurological symptoms and were attributed to the uniquely defined areas of impaired blood supply. StBr concept may be useful for an integrated radiological CT-based assessment of suspected stroke cases or can be included into computer-aided tools to optimise the evaluation of stroke site and its extent. These data in turn are appropriable for further diagnosis, predicting the therapeutic outcome as well as for patients' qualification for an appropriate form of reperfusion therapy. The usefulness of StBr was illustrated in the case studies. (Folia Morphol 2017; 76, 4: 568-573)
\end{abstract}

Key words: cerebral arteries, stroke, ischaemic stroke, brain ischaemia, cerebral haemorrhage, computed tomography

\section{INTRODUCTION}

Stroke is one of the heaviest and the most devastating brain diseases. According to World Health Organisation reports, in 2012 it was second, after ischaemic heart disease, cause of death in the world, responsible for 6.7 million deceases. It is essential to remember that stroke can happen to anyone at any time, regardless of race, sex or age. In the United States of America one episode of stroke occurs every $40 \mathrm{~s}$, taking one life approximately every $4 \mathrm{~min}$.

Stroke is equivalent to the loss of brain function caused by a disturbance in the blood supply to the brain. Two main types of stroke are ischaemic stroke and haemorrhagic stroke. Ischaemic stroke constitutes more than $80 \%$ of all strokes [4]. Within recent years a huge step forward in the treatment of ischaemic stroke was made. The introduction of

Address for correspondence: Prof. B. Ciszek, Department of Descriptive and Clinical Anatomy, Centre of Biostructure Research, Medical University of Warsaw, ul. Chałubińskiego 5, 02-004 Warszawa, Poland, tel: +48 2262952 83, fax: +48 2262952 83, e-mail: bjciszek@o2.pl 
tissue plasminogen activator (tPA) and endovascular revascularisation therapies have led to improved patient outcomes following successful recanalisation of an occluded intracranial vessel [5]. As shown in many randomised and placebo-controlled trials $[6,10,11]$, thrombolysis is the most effective treatment of ischaemic stroke when introduced up to $3 \mathrm{~h}$ after the onset. The main goals of neuroimaging are concentrated on the confirmation of stroke (excluding common ischaemic stroke mimics), exclusion of haemorrhagic foci (the most important contraindication for revascularisation therapy) and proper localisation of a site and extent of the vascular occlusion, which is based on recognition of anatomically defined ischaemic areas. However, the identification of ischaemic changes is not straightforward. At a hyperacute stage (less than $3 \mathrm{~h}$ from the onset) the hypodense changes in non-contrast computed tomography (CT) are barely perceptible or even invisible. This is because CT shows well a necrosis of the brain tissue, while at the hyperacute stage the process of tissue destruction is still ongoing, thus only some indirect early ischaemic signs (e.g. a hyperdense vessel) can be observed. Unfortunately, these early signs are characterised by the lack of sensitivity and are observer dependent. Consequently, the selection of possible anatomical regions of ischaemic stroke is difficult at early stage.

In this paper we describe the method of selection and evaluation of brain areas which could be useful for localisation of stroke regions by specialist in Emergency Stroke Unit. We suggest to call these assigned tissue blocks Stroke Bricks (StBr). Currently proposed scheme of brain division is limited to the supratentorial space of the brain. StBr in the infratentorial space will be the topic of separate paper.

\section{MATERIALS AND METHODS}

The proposed StBr areas are associated with clinical symptoms observed during the ongoing ischaemia. They cover the grey and white matter and the areas of fluid space and constitute specific segments of the brain. Within the cerebral hemisphere StBr respects the main areas of vascularisation.

The definition of successive $\mathrm{StBr}$ regions is as follows. Area A covers the medial part of the hemisphere from the area subcallosa to the parieto-occipital sulcus. It is laterally bordered by the sagittal plane running at the level of the superior frontal sulcus. Within this area, A1 segment extends from the frontal pole to the precentral sulcus. A2 segment corresponds to the paracentral lobule. Backward is the A3 segment, which includes precuneus and superior parietal lobule. Area $\mathrm{P}$ includes the rear part of the medial surface of the hemisphere with the occipital pole and the mediobasal part of the temporal lobe with the parahippocampal gyrus without the uncus, which is highlighted as an area UA. The area M covers superolateral part of the cerebral hemisphere between areas $A$ and $P$. In the upper part of area $M$ we distinguish segments: M4 - between area $A$ and precentral gyrus, M5 - including the precentral and postcentral gyri (sensorimotor area) and M6 - located backward to the postcentral sulcus and corresponding to the inferior parietal lobule. Below the M4 segment, an M1 segment is located and it represents lower part of the frontal lobe from the upper border of the insula. Segments M2 and M3 constitute the anterior and posterior part of the temporal lobe and are divided at the level of posterior margin of insula. Medial delineation of the M4-M6 segments constitutes an area corona radiata (CR), covering the oval part of the white matter in the depth of the hemisphere. Below area $\mathrm{CR}$ we distinguished a region of basal ganglia surrounding the internal capsule, described as area BGIC. Area $T$ - the thalamus is located medially to BGIC.

We used normalised CT template (based on healthy individuals at the age corresponding to that commonly seen in stroke -65 years in average) from SPM Toolbox [8] for model StBr regions delineation. Template creation and normalisation procedure was described in details in Rorden et al. [8]. The segmentation of StBr regions was made manually by neurologist, based on visible anatomical structures (commonly observed on brain CT images). Regions outlines (with transparent colour overlays) were prepared using ImageJ software. Model StBr regions are presented on Figure 1A, B. The common neurological symptoms and corresponding $\mathrm{StBr}$ regions are presented in Table 1.

\section{ILLUSTRATIVE CASES}

We present three cases in order to illustrate the example application of StBr in real clinical situations. Each of 3 patients underwent an initial CT scan immediately after admission (up to $4.5 \mathrm{~h}$ after the onset of stroke). Secondary control imaging (CT or magnetic resonance imaging $[\mathrm{MRI}]$ ) was performed after few days (always more than $24 \mathrm{~h}$ from the stroke symp- 


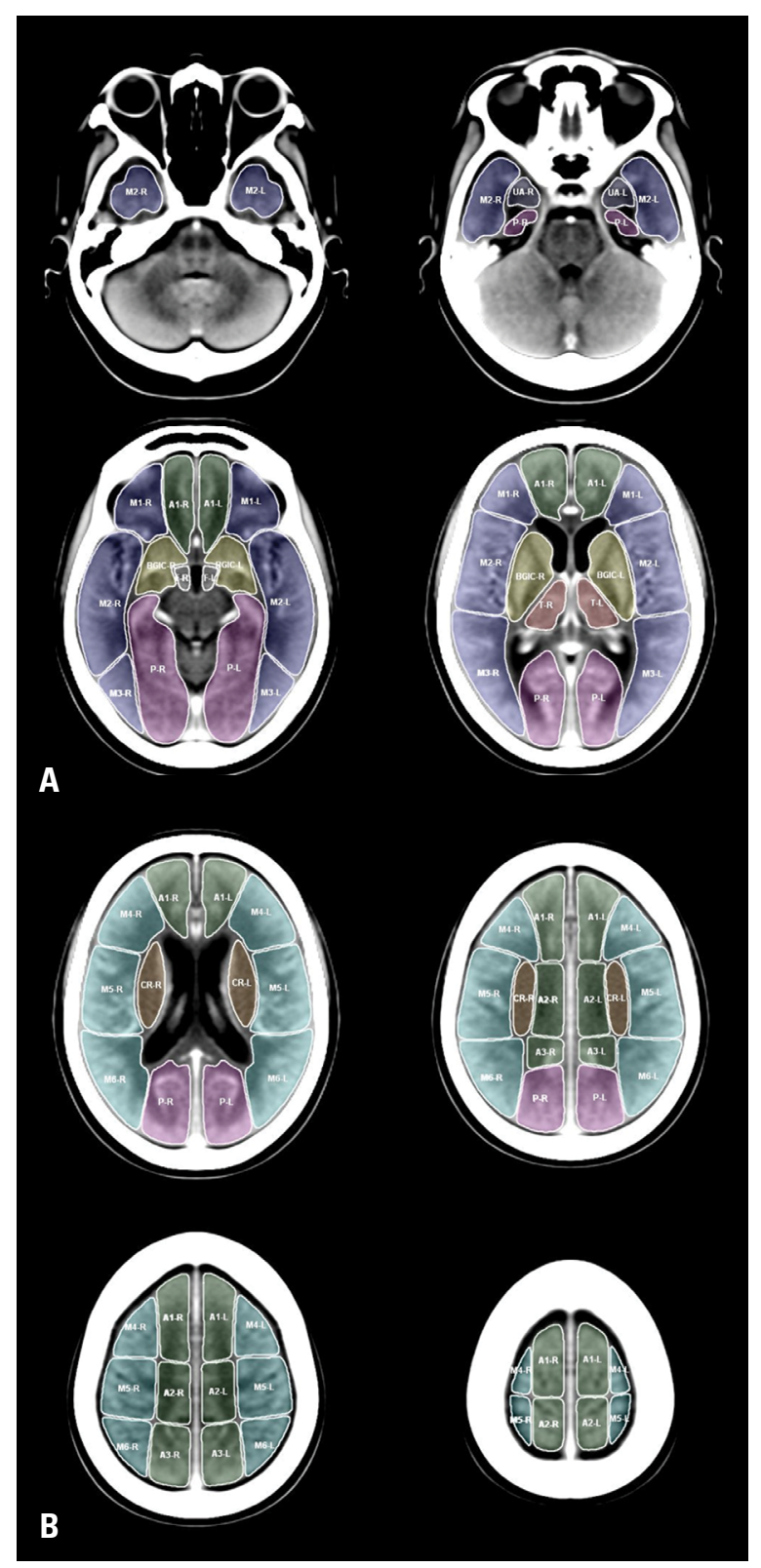

Figure 1. A, B. Stroke Bricks - proposed brain regions division scheme presented in selected slices from normalised computed tomography template.

toms onset, when the stroke is clinically confirmed). The diagnosis of stroke and its extent was subsequently confirmed by a radiologist.

\section{Case 1}

A 63-year-old woman with the history of hypertension and hyperthyroidism was admitted to the Emergency Department due to sudden right hemiparesis. Neurological examination revealed faint right-sided hemiparesis. NIHS score was 2 . NCCT scan ( $4.5 \mathrm{~h}$ from the stroke symptoms onset)
Table 1. Common neurological symptoms and corresponding Stroke Bricks segments

\begin{tabular}{lc}
\hline Symptom & Regions \\
\hline Gaze palsy or total gaze paresis & $\mathrm{M} 1, \mathrm{M} 4$ \\
Expressive (motor) aphasia & $\mathrm{M} 1, \mathrm{M} 4$ \\
Hemineglect & $\mathrm{A} 3, \mathrm{M} 6$ \\
Sensory aphasia & $\mathrm{M} 3, \mathrm{M} 6$ \\
Disorientation & $\mathrm{A} 1, \mathrm{M} 1, \mathrm{M} 4$ \\
Central facial nerve palsy & $\mathrm{M} 5, \mathrm{CR}, \mathrm{BGIC}$ \\
Central hypoglossal nerve palsy & $\mathrm{M} 5, \mathrm{CR}, \mathrm{BGIC}$ \\
Homonymous hemianopsia & $\mathrm{P}, \mathrm{M} 2, \mathrm{M} 3$ \\
Upper limb paresis & $\mathrm{M} 5, \mathrm{CR}, \mathrm{BGIC}$ \\
Lower limb paresis & $\mathrm{A} 2, \mathrm{CR}, \mathrm{BGIC}$ \\
Upper limb sensory loss & $\mathrm{M} 5, \mathrm{CR}, \mathrm{BGIC}, \mathrm{T}$ \\
Lower limb sensory loss & $\mathrm{A} 2, \mathrm{CR}, \mathrm{BGIC}, \mathrm{T}$ \\
Face hemisensory loss & $\mathrm{M} 5, \mathrm{CR}, \mathrm{T}$ \\
Dysarthria & $\mathrm{A} 1, \mathrm{M} 1, \mathrm{M} 4, \mathrm{BGIC}$ \\
\hline
\end{tabular}

showed no significant changes. Based on neurological symptoms an assumption of ischaemic stroke in the left-side regions $\mathrm{A} 2, \mathrm{M} 5, \mathrm{BGIC}$ and $\mathrm{CR}$ was made. Visual suggestion of possible regions of ischaemia is presented in selected slices from initial NCCT examination (Fig. 2). Secondary MRI imaging (performed 5 days after stoke onset) revealed brain ischaemia in the region of left corona radiata (CR-L) which is consistent with the primary assumption (Fig. 3 ).

\section{Case 2}

A 63-year-old man with no prior medical history was admitted to the Emergency Department due to a sudden left hemiparesis. Neurological examination revealed partial gaze palsy to the right, partial left hemianopia, central left facial nerve palsy, severe leftsided hemiparesis, dysarthria as well as Babinsky's sign on the left. NIHS score was 14. NCCT scan (4.5 h from the stroke symptoms onset) showed no significant changes. Based on neurological symptoms an assumption of ischaemic stroke in the right-side regions $A 1$, A2, M1, M2. M3, M4, M5, P, BGIC and CR was made. Visual suggestion of possible regions of ischaemia is presented in selected slices form initial NCCT examination (Fig. 4). Secondary CT imaging (performed 1.5 day after stoke onset) confirmed a primary assumption of large ischaemia in the region supplied by the right internal carotid artery (Fig. 5), covering brain cortex (M1-M5 and A1-A2) without deep brain structures. 


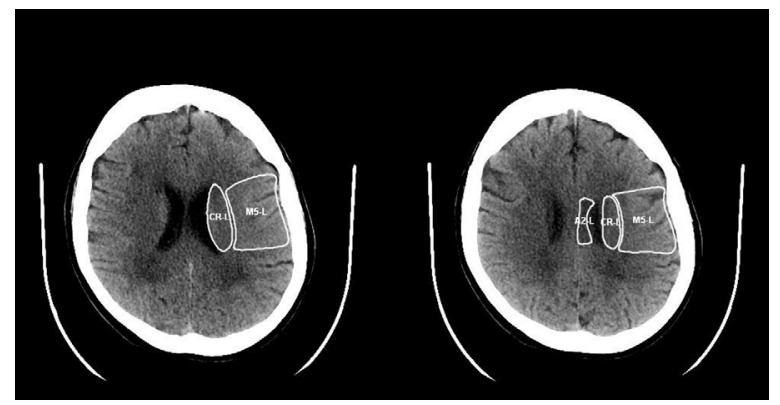

Figure 2. Case $1-$ a visual suggestion of possible locations of stroke in Stroke Bricks regions A2-L, CR-L, M5-L presented in selected slices from NCCT examination.

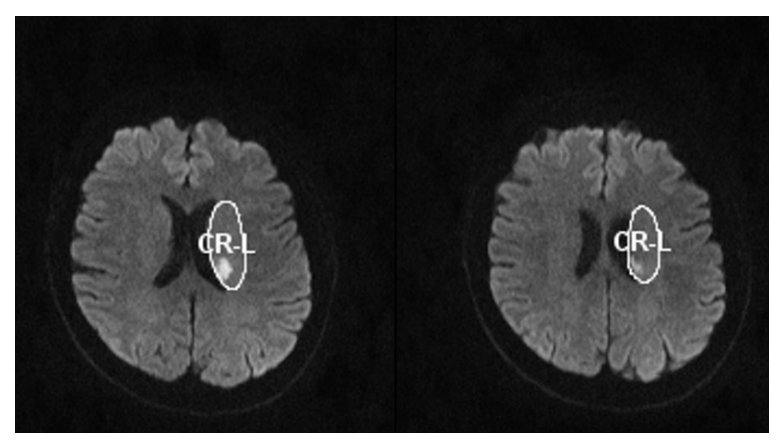

Figure 3. Case 1 - confirmation of stroke localisation on corresponding slices from secondary imaging (magnetic resonance imaging). Brain ischaemia revealed in the left corona radiata area CR-L.

\section{Case 3}

A 42-year-old man with vasculitis, during glucocorticosteroid treatment, was admitted to the Emergency Department due to sudden speech disturbance and right hemiparesis, faint right hemisensory loss. Neurological examination revealed global aphasia, forced gaze palsy to the left side, right abducens (sixth) nerve palsy and severe right-sided hemiparesis. NIHS score was 16 . NCCT scan $(0.75 \mathrm{~h}$ from the stroke symptoms onset) showed no significant changes. An ischaemic stroke in the left-side regions $A 2, M 1, M 3$, M4, M5, M6, BGIC, CR and T was considered based on neurological symptoms (Fig. 6). Secondary CT imaging (performed 1.5 day after stoke onset) confirmed a diagnosis of an ischaemic stroke within suggested locations (Fig. 7). The absorbed region turned to be limited to upper branch of MCA (M1-L), internal capsule (BGIC-L) and corona radiata (CR-L).

\section{DISCUSSION}

The proposed allocation of brain areas of interest was inspired by the conception of ASPECTS used in a quantitative evaluation of early ischaemic changes within the middle cerebral artery territory on NCCT [1]. We decided to extend and adapt this idea to the entire content of the supratentorial space. In contrary to the ASPECTS we developed precise boundaries of the proposed areas. This is also due to the fact, that within the classically designated areas of vascularisation a large individual anatomical and functional variability is observed $[2,9]$. We also included fluid spaces which are necessary for an analysis of a subarachnoid haemorrhage.

The presented division scheme is arbitrary and only quasi-anatomical but it stays in relation to the areas of vascularisation. Its idea was to create a simple topographic division of brain tissue useful for objectified rapid radiological assessment, computer-aided diagnosis and clinical decision support systems in the diagnostics of ischaemic stroke. A specific set of neurological symptoms may indicate $\mathrm{StBr}$ regions - locations of the source of these symptoms. Selected StBr segments show the area occupied by stroke with a relation to regions of vascularisation. The presented system of $\mathrm{StBr}$ segments can be also useful in the assessment of the extent of ischaemia, thus it might improve the accuracy of therapeutic decision for reperfusion therapy. The initial implementation of StBr was integrated into the computerised system supporting diagnosis of ischaemic stroke in emergency [7].

The concept of $\mathrm{StBr}$ can be extended of a numerical scale reflecting the extent of stroke. The final score can be achieved by the summation of affected $\mathrm{StBr}$, as the clinical symptoms correspond with assigned brain regions. Therefore, the area of stroke may be defined with a value ranging from 0 to 13 (where 13 is a total number of all possible regions affected in one hemisphere). A total sum of stroke-affected areas may provide an initial description of the extent of tissue destruction and thus may be used for further stratification and prognosis. Similarly, an assessment of the extent of a subarachnoid haemorrhage and an intracerebral haemorrhage can be carried out basing on the proposed system.

\section{CONCLUSIONS}

Recent advances introduced conception of prehospital thrombolysis realised in a form of stroke emergency mobile unit (STEMO). The STEMO ambulance is equipped with a computed tomographic scanner with a point-of-care laboratory and telemedicine connection. The evaluation of images acquired in the STEMO board is made on call by neuroradiologist using a teleradiology connection [3]. Described examples 


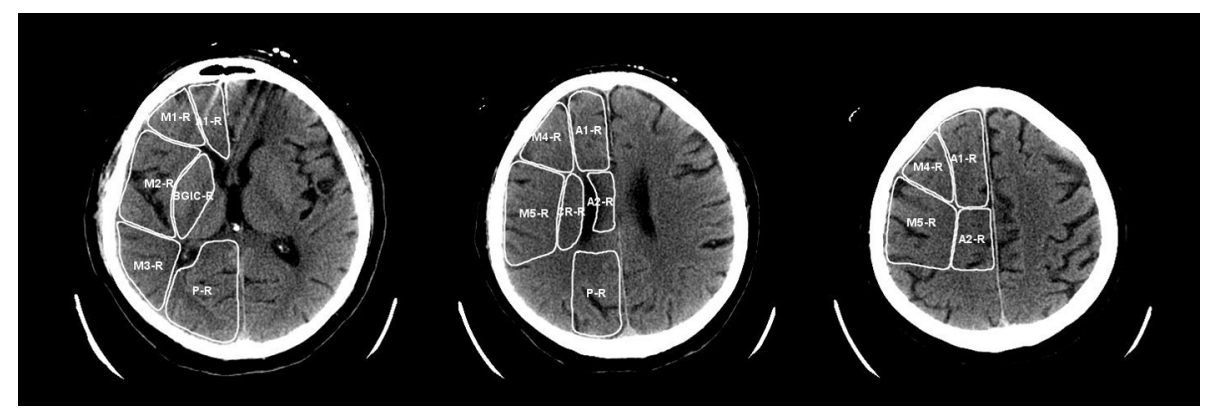

Figure 4. Case 2 - a visual suggestion of possible locations of stroke in Stroke Bricks regions A1-R, A2-R, M1-R, M2-R, M3-R, M4-R, M5-R, $\mathrm{P}-\mathrm{R}, \mathrm{BGIC}-\mathrm{R}$ and CR-R presented in selected slices from NCCT examination.

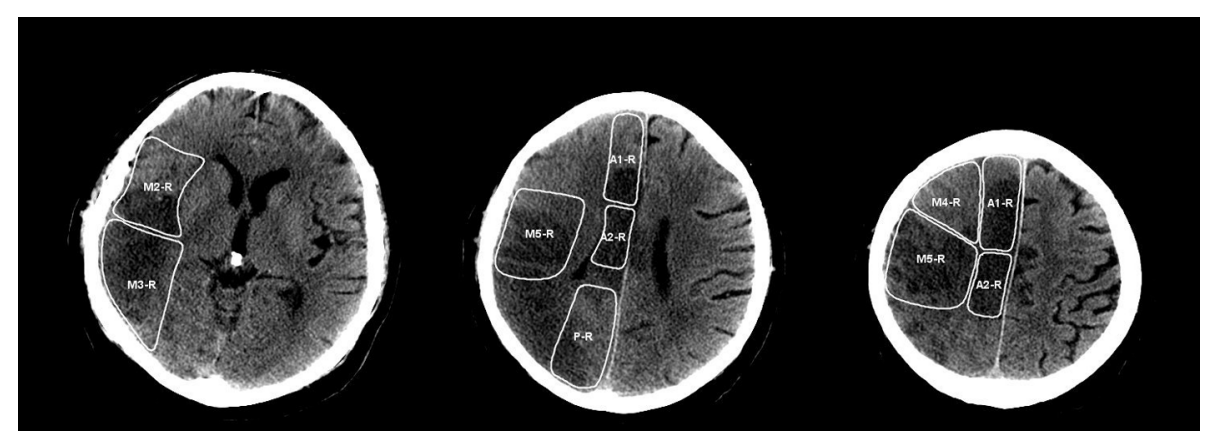

Figure 5. Case 2 - confirmation of stroke localisation in brain cortex (affected right-side regions M1-M5 and A1-A2) shown in corresponding slices from secondary computed tomography imaging.

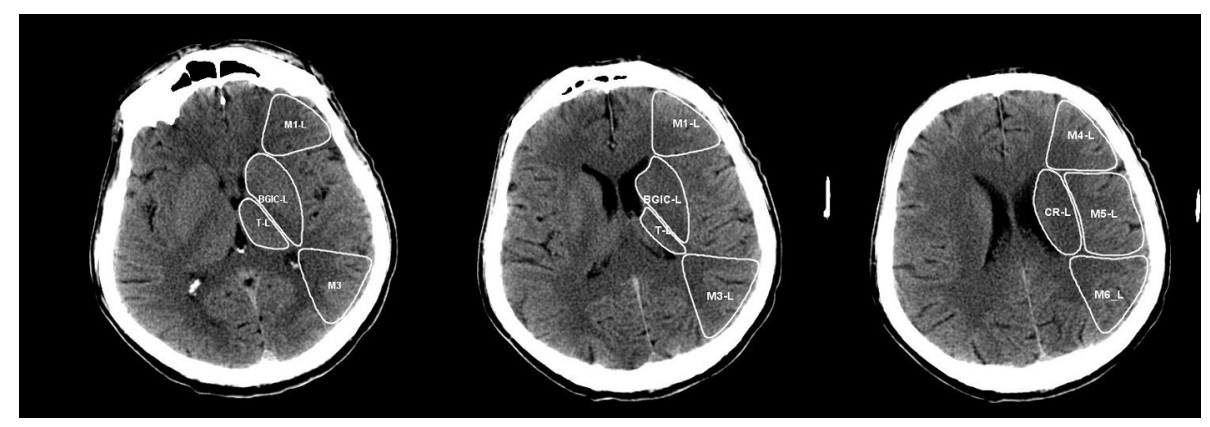

Figure 6. Case 3 - a visual suggestion of possible locations of stroke in Stroke Bricks regions A2-L, M1-L, M3-L, M4-L, M5-L, M6-L, T-L, BGIC-L and CR-L presented in selected slices from NCCT examination.

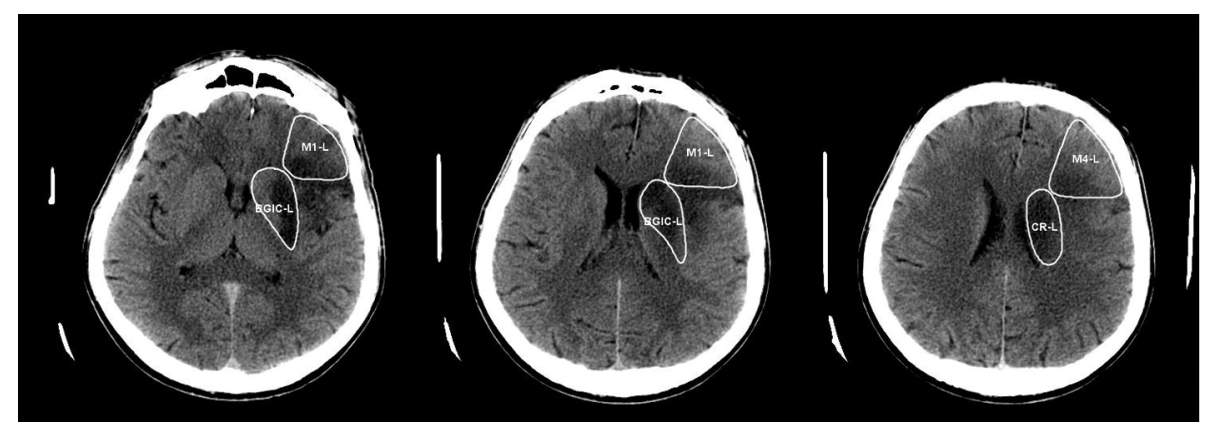

Figure 7. Case 3 - confirmation of stroke localisation in corresponding slices from secondary computed tomography imaging. Brain ischaemia revealed in the left BGIC, CR and M1 region. 


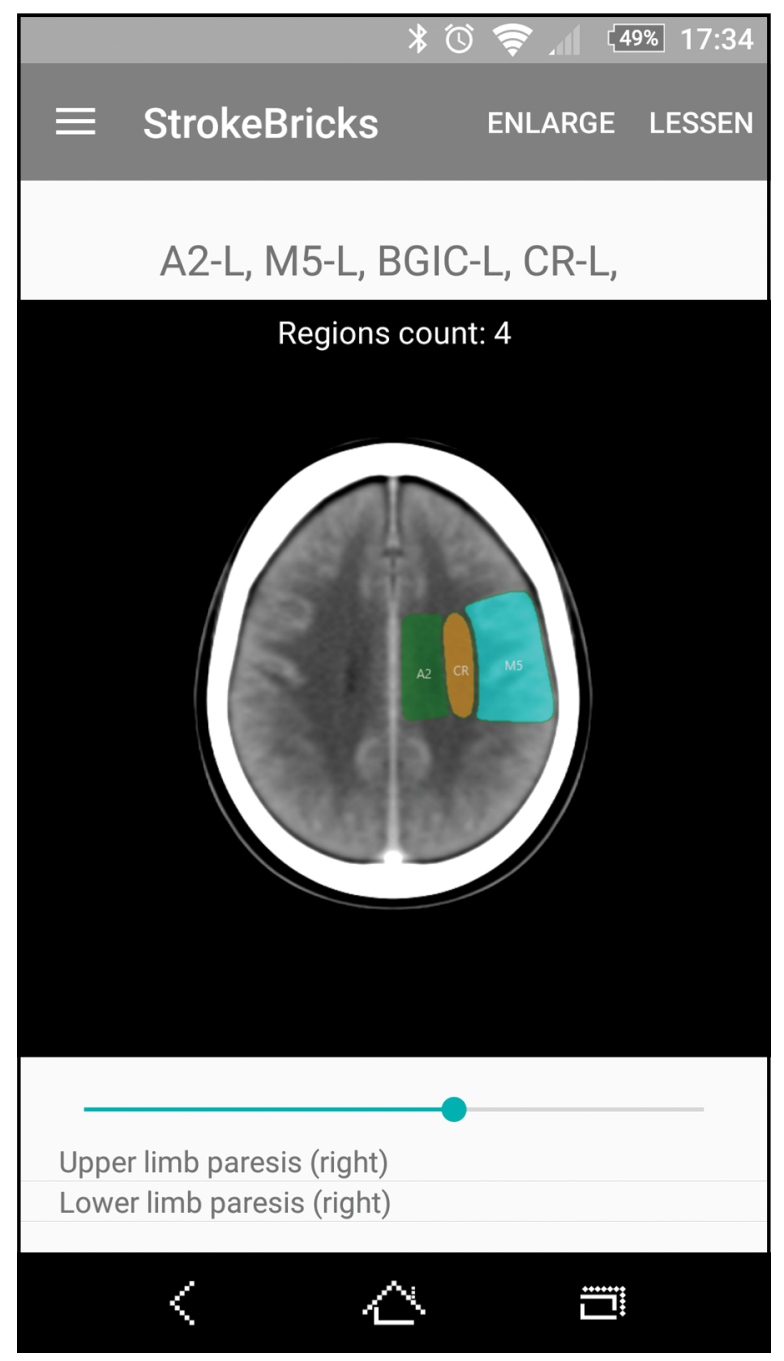

Figure 8. Prototype version of mobile Stroke Bricks application.

illustrate the accuracy of StBr, which quickly suggests the potential location of stroke, and may be a useful additional tool in mobile teleradiology scenario.

The $\mathrm{StBr}$ visual suggestion is usually broader in range than the actual ischaemic region but always includes the actual one. It may help the neurologist and the radiologist in the assessment of the location and extent of stroke by providing essential information for further therapeutic process in the mobile or standard emergency units. The use of StBr may improve patients' evaluation and enrolment to the appropriate way of treatment with special attention to early pharmacological intravenous thrombolysis or endovascular stroke therapies (e.g. mechanical thrombectomy).

Prototype version of mobile $\mathrm{StBr}$ application is presented on Figure 8. It could help with naming and visualising the regions connected with common neurological stroke symptoms.

\section{Acknowledgements}

This publication was funded by the National Science Centre (Poland) based on the decision DEC-2011/03/B/ST7/03649.

\section{REFERENCES}

1. Barber PA, Demchuk AM, Zhang J, et al. Validity and reliability of a quantitative computed tomography score in predicting outcome of hyperacute stroke before thrombolytic therapy. ASPECTS Study Group. Alberta Stroke Programme Early CT Score. Lancet. 2000; 355(9216): 1670-1674, indexed in Pubmed: 10905241.

2. Blackburn K, Labranche L, Kalmey J, et al. A case of a single intracranial vertebral artery and cerebral infarct. Folia Morphol. 2017; 76(1): 134-138, doi: 10.5603/fm. a2016.0033.

3. Ebinger M, Kunz A, Wendt $M$, et al. Effects of golden hour thrombolysis: a prehospital acute neurological treatment and optimization of medical care in stroke (PHANTOM-S) substudy. JAMA Neurol. 2015; 72(1): 25-30, doi: 10.1001/jamaneurol.2014.3188, indexed in Pubmed: 25402214.

4. El-Koussy M, Schroth G, Brekenfeld C, et al. Imaging of acute ischemic stroke. Eur Neurol. 2014; 72(5-6): 309-316, doi: 10.1159/000362719, indexed in Pubmed: 25323674.

5. Heit JJ, Wintermark M. Imaging selection for reperfusion therapy in acute ischemic stroke. Curr Treat Options Neurol. 2015; 17(2): 332, doi:10.1007/s11940-014-0332-3, indexed in Pubmed: 25619536.

6. Sandercock P, Wardlaw JM, Lindley RI, et al. IST-3 collaborative group. The benefits and harms of intravenous thrombolysis with recombinant tissue plasminogen activator within $6 \mathrm{~h}$ of acute ischaemic stroke (the third international stroke trial [IST-3]): a randomised controlled trial. Lancet. 2012; 379(9834): 2352-2363, doi: 10.1016/S0140-6736(12)60768-5, indexed in Pubmed: 22632908.

7. Przelaskowski A, Sobieszczuk E, Jóźwiak R, et al. Integrated System for Clinical Decision Support in Emergency Stroke Care. Info Technol Med. 2016: 457-473, doi: 10.1007/9783-319-39796-2 38.

8. Rorden C, Bonilha L, Fridriksson J, et al. Age-specific CT and MRI templates for spatial normalization. Neuroimage. 2012; 61(4): 957-965, doi:10.1016/j.neuroimage.2012.03.020, indexed in Pubmed: 22440645.

9. Szarmach A, Winklewski PJ, Halena G, et al. Morphometric evaluation of the delayed cerebral arteries response to acetazolamide test in patients with chronic carotid artery stenosis using computed tomography angiography. Folia Morphol. 2017; 76(1): 10-14, doi: 10.5603/ FM.a2016.0034, indexed in Pubmed: 27830888.

10. Wardlaw J, Murray V, Berge $E$, et al. Recombinant tissue plasminogen activator for acute ischaemic stroke: an updated systematic review and meta-analysis. Lancet. 2012; 379(9834): 2364-2372, doi: 10.1016/s01406736(12)60738-7.

11. Wardlaw J, Murray V, Berge E, et al. Thrombolysis for acute ischaemic stroke. Cochrane Database Syst Rev. 2014; 29(7): CD000213, doi:10.1002/14651858. cd000213.pub3. 